\title{
POPULATIONS OF MASSIVE X-RAY BINARIES
}

\author{
Ignacio Negueruela ${ }^{1}$ \\ RESUMEN
}

La población de binarias masivas de rayos X en una galaxia nos informa sobre su historia reciente de formación estelar. Sin embargo, es necesario un conocimiento más profundo de la población de estos sistemas en la Vía Láctea y sus mecanismos de formación para poder explotar esta información.

\section{ABSTRACT}

The population of massive X-ray binaries in a galaxy can provide important constraints on its recent history of star formation. However, further knowledge of the Galactic population and formation mechanisms is needed before deeper inferences can be made for other galaxies.

\section{Key Words: BINARIES: CLOSE — PULSARS: GENERAL - X-RAYS: BINARIES}

\section{INTRODUCTION}

Massive X-ray Binaries (MXBs) evolve from binary systems containing two massive stars. They are hence necessarily young and trace recent star formation. Traditionally, two main groups of MXBs have been recognised. One contains a Be star and a neutron star. These Be/X-ray binaries (BeXs) are believed to descend from moderately massive binaries $\left(M_{\text {total }} \approx 20-30 M_{\odot}\right)$. In Supergiant X-ray Binaries (SXBs), the mass donor is a relatively evolved massive star, with luminosity class I or II. SXBs are thought to evolve from more massive binaries.

In recent years, the number of MXBs known in the Milky Way and the Magellanic Clouds has grown so much that we can now treat their populations as statistically significant samples that can be compared. For example, as SXBs are expected to be younger than BeXs, they sample stellar populations of slightly different ages. The Milky Way and the LMC show similar relative fractions of SXBs and BeXs (around $2 / 3$ are BeXs). This coincidence may be taken as an indication that selection effects are not veiling our view of these populations. The ratio should be representative of more or less steady star formation (Negueruela \& Coe 2002).

The situation in the SMC is very different. For a start, the number of MXBs known is an order of magnitude higher than expected from a simple extrapolation of the LMC values, providing strong confirmation of a higher rate of star formation. Moreover, out of 37 confirmed X-ray pulsars in the SMC, only one (SMC X-1) is an SXB. The weak winds of B-type supergiants at low metallicity are likely to result in much lower X-ray luminosities for SMC

\footnotetext{
${ }^{1}$ Universidad de Alicante, Spain.
}

SXBs than for their Galactic counterparts. However, the absence of detections even in deep surveys suggests that SXBs are indeed very scarce in the SMC. which could be explained if the population of the SMC is the result of a large burst of star formation that ended $\sim 10$ Myr ago.

\section{CLASSES OF MXBS IN THE MILKY WAY}

The class of BeX binaries is simply defined by the fact that the mass donor is an emission-line star. BeXs in the Milky Way display a variety of properties. There have been several attempts at finding commonalities that may define classes of BeXs, in the hope that this division will give us information about physical mechanisms.

While most BeX systems are transients displaying X-ray outbursts (see Okazaki \& Negueruela 2001. for our current understanding of these issues), a small group seems to show much less X-ray variability. Based on a sample of four pulsars (listed in the upper panel of Table 1), Reig \& Roche (1999) propose the following characteristics differentiating "persistent" BeX from BeX transients:

(1) Relatively long pulse periods $>200 \mathrm{~s}$.

(2) Persistent, low luminosity X-ray emission (ミ $10^{35} \mathrm{erg} \mathrm{s}^{-1} \mathrm{~cm}^{-2}$ ) with small fluctuations.

(3) The X-ray spectrum presents a low cut-off energy $(\sim 4 \mathrm{keV}$, compared to $10-20 \mathrm{keV}$ in transients) and no dependence on intensity.

(4) Absence of, or very weak, iron line at $6.4 \mathrm{keV}$.

Other X-ray pulsars have beenl proposed to be members of this class (second panel of Table 1 ). Among them, AX J0049.5-7323 and AX J0103-72.2 are BeX systems in the SAC sharing many of the defining characteristics, but the Galactic candidates have no optical counterpart and there is a small 
TABLE 1

PERSISTENT BrX AND CANDIDATES.

\begin{tabular}{lr}
\hline Object & $P_{\mathrm{s}}(\mathrm{s})$ \\
\hline X Per & 835 \\
RX J0146.9+6121 & 1404.2 \\
RX J0440.9+4431 & 202.5 \\
RX J1037.5-5647 & 862 \\
\hline AX J0049.5-7323 & 756 \\
AX J0103-722 & 345 \\
1SAX J1452.8-5949 & 437.4 \\
AX J1749.2-2725 & 220.4 \\
AX J1700-419 & 714.5 \\
\hline
\end{tabular}

TABLE 2

Be/X-RAY TRANSIENTS WITH LOW ECCENTRICITIES.

\begin{tabular}{lrr}
\hline Object & $P_{\text {orb }}$ (days) & $e$ \\
\hline GS 0834-430 & $105.80 \pm 0.40$ & $<0.17$ \\
XTE J15.3-568 & $75.56 \pm 0.25$ & $<0.03$ \\
KS 1947+300 & $40.42 \pm 0.02$ & $<0.04$ \\
2S 1553-542 & $30.60 \pm 2.20$ & $<0.09$ \\
\hline
\end{tabular}

chance that they are really cataclysmic binaries. More recently, after the determination of the orbital parameters of X Per $\left(e=0.11, P_{\text {orb }}=250 \mathrm{~d}\right)$ by Delgado-Martí et al. (2001), Pfahl et al. (2002) proposed this object as the prototype for a new class of MXBs characterised by long $\left(P_{\text {orb }}>30 \mathrm{~d}\right.$ ) orbital periods and low eccentricities. As the long orbital periods imply that tidal circularisation cannot have occurred after the supernova explosion that created the neutron star, the low eccentricities are primordial: the systems must have formed in a supernova explosion not accompanied by the velocity kicks that are necessary to explain the eccentric orbits of most BeX transients (van den Heuvel \& van Paradijs 1997). The class is defined by X Per and the four loweccentricity BeX transients listed in Table 2. There are, however, strong reasons to believe that all the "persistent" BeX systems, and also some transients (like A $1118-615, P_{\mathrm{s}}=407 \mathrm{~s}$ ), have wide orbits and low eccentricities (cf. Okazaki \& Negueruela 2001). Therefore the class of $\mathrm{BeX}$ with near-circular orbits (from now on, low-e $\mathrm{BeX}$ ) cuts across the distinction between persistent and transient $\mathrm{BeX}$. Podsiadlowski et al. (2003) have suggested that the existence of the class of low-e $\mathrm{BeX}$ can be naturally explained by a bimodal distribution in the velocity kicks, due to dif- ferent modes of explosion, with neutron stars formed in an electron-capture supernova receiving a very small kick. Therefore the class of low-e BeX would be justified by a physical reason and hence a useful instrument for furthering our knowledge of MXB formation mechanisms. One word of caution, however. is necessary. Two of the transients in Table 2, XTE J1543-568 and KS 1947+300, have essentially circular orbits. While the low-e orbit of X Persei may be explained by a supernova explosion without kick (all induced eccentricity is due to mass loss), XTE J1543-568 and KS 1947+300 must have been born with basically no eccentricity, meaning that either no mass was lost in the supernova explosion or that the kick velocity exactly balanced the impulse due to mass loss. Both options are essentially impossible. According to all models, tidal circularisation in such wide orbits can only occur when the star enters the supergiant phase (as a matter of fact, SXBs have effectively circular orbits). However, the optical counterpart of KS $1947+300$ is known (Negueruela et al. 2003) and it is a main-sequence star. New intermediate resolution spectra have been obtained with the 4.2-m WHT (in La Palma) equipped with the ISIS spectrograph and confirm a spectral type O9.5Ve. XTE J1543-568 has no known counterpart, but its behaviour is also typical of BeX transients. Therefore the mystery of these essentially circular systems, whose existence is incompatible with all current models, must be solved before we can effectively use the class of low-e $\mathrm{BeX}$ to constrain our knowledge of possible MXB formation paths.

The author is a researcher of the programme Ramón y Cajal, funded by the Spanish MCyT and the University of Alicante. This research is partially supported by the Spanish MCyT under grants AYA2002-00814 and ESP2001-4541-PE.

\section{REFERENCES}

Delgado-Martí, H., Levine, A.M., Pfahl, E., \& Rappaport, S.A. 2001, ApJ 546, 455

van den Heuvel, E.P.J., \& van Paradijs, J. 1997, ApJ 483, 399

Negueruela, I., \& Coe, M.J. 2002, A\&A 385, 517

Negueruela, I., Israel, G. L., Marco, A., et al. 2003, A\&A 397, 739

Okazaki, A.T., \& Negueruela, I. 2001, A\&A 377, 161

Pfahl, E., Rappaport, S. Podsiadlowski, P., \& Spruit, H. 2002, ApJ 574, 364

Podsiadlowski, Ph., Langer, N., Poelarends, A.J.T., et al. 2003, ApJ, in press

Reig, P., \& Roche, P. 1999, MNRAS 306, 100 